\title{
Design and Stability of an On-Orbit Attitude Control System Using Reaction Control Thrusters
}

Robert A. Hall

CRM Solutions, Inc., Jacobs ESSSA Group, Huntsville Al, 35812
Steven Hough Dynamic Concepts, Inc., Jacobs ESSSA Group, Huntsville Al, 35812
Carolina Orphee

NASA Marshall Space Flight Center, Huntsville Al, 35812
Keith Clements Engineering Research and Consulting, Inc, Jacobs ESSSA Group, Huntsville Al, 35812

Guidance, Navigation, and Control Conference, AIAA SciTech 2016 San Diego, California, January 4-8, 2016 


\section{Overview}

- NASA is providing preliminary design and requirements for the Space Launch System Exploration Upper Stage (EUS).

- The EUS will provide upper stage capability for vehicle ascent as well as onorbit control capability.

- Requirements include performance of on-orbit burn to provide Orion vehicle with escape velocity.

- On-orbit attitude control is accommodated by a on-off Reaction Control System (RCS).

- Paper provides overview of approaches for design and stability of an attitude control system using a RCS.

- Draws heavily from research and development in support of Space Shuttle and Space Station programs. Includes pitfalls and lesson's learned from flight experience. 


\section{Paper Summary}

- Vehicle Attitude Dynamics and Phase Plane Control

- Phase Plane Stability and Filter Design

- Jet Selection

- Maneuver/Steering Algorithms

- Thruster Hardware Specifications 


\section{Vehicle Attitude Dynamics and Phase Plane Control}

- Attitude Dynamics are summarized:

$$
\begin{aligned}
& J_{1} \omega_{1}=\left(J_{2}-J_{3}\right) \omega_{2} \omega_{3}+T_{1_{\Delta z z}}+u_{1} \\
& J_{2} \dot{\omega}_{2}=\left(J_{3}-J_{1}\right) \omega_{1} \omega_{3}+T_{2_{s x t}}+u_{2} \\
& J_{3} \dot{\omega}_{3}=\left(J_{1}-J_{2}\right) \omega_{1} \omega_{2}+T_{3_{c x t}}+u_{3} \\
& \eta=\cos \left(\frac{\varphi}{2}\right) \quad \dot{\eta}=-\frac{1}{2} \varepsilon^{T} \hat{\omega} \\
& \hat{\varepsilon}=\hat{a} \sin \left(\frac{\varphi}{2}\right) \quad \dot{\hat{\varepsilon}}=\frac{1}{2}\left(\hat{\varepsilon}^{x}+\eta I\right) \hat{\omega} \\
& \Lambda_{f g}=\left[\begin{array}{ll}
\eta & \hat{\varepsilon}
\end{array}\right]
\end{aligned}
$$

- Phase Plane Design Examples are Provided:

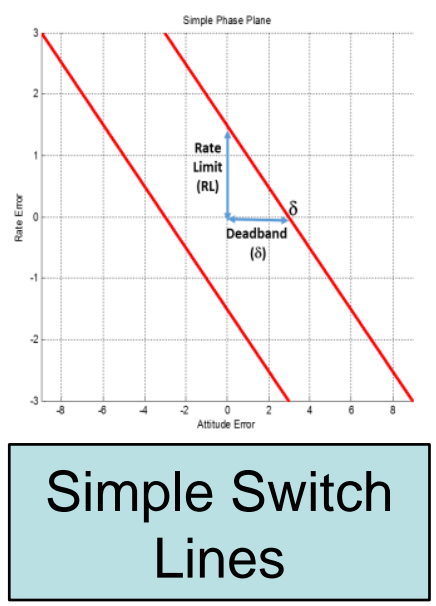

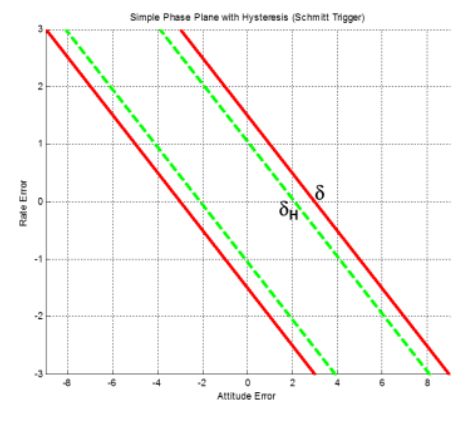

Hysteresis Added
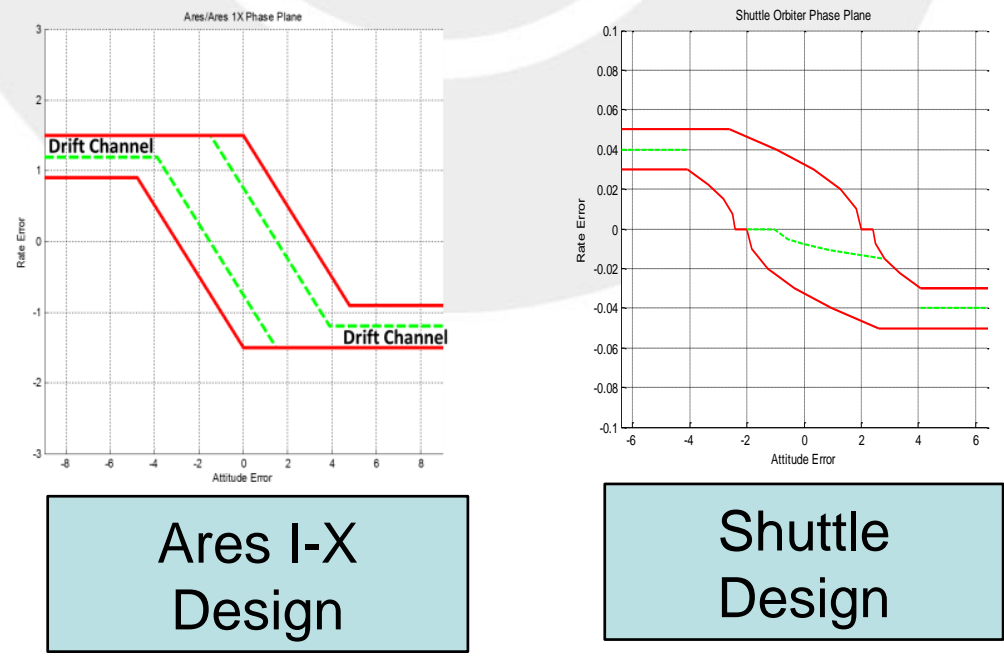


\section{Phase Plane Stability and Filter Design}

- Phase Plane control designs are nonlinear, hence traditional linear design approaches are generally not available.

- Paper presents RCS filter design and phase plane stability approaches based on research performed on the Space Shuttle and Space Station programs

- Stability margin design goals are provided:

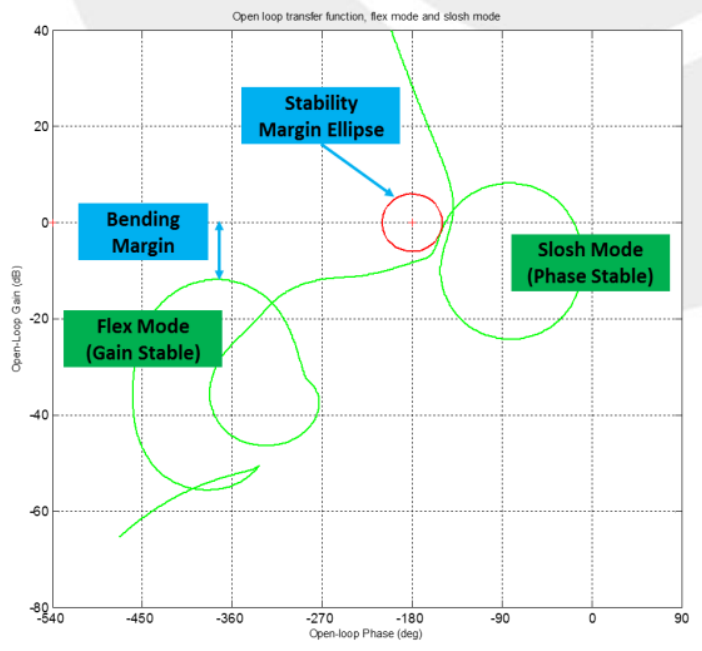




\section{Phase Plane Stability (continued)}

- Paper describes approaches to derive a linear representation of the nonlinear system, concentrating on describing functions.

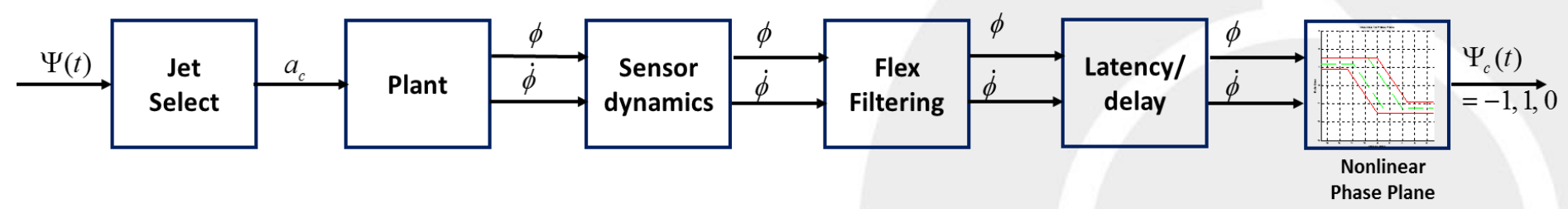

- Phase plane is converted into an equivalent PD controller with a relay:

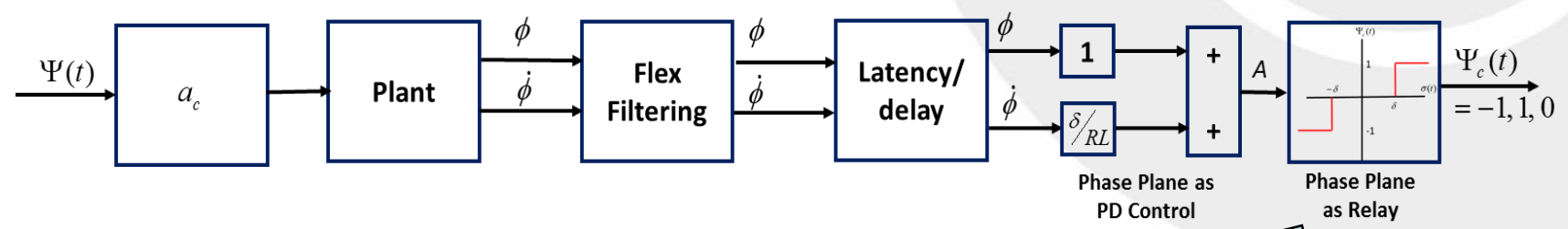

- Relay is modeled by a describing function.

$N(A)=0$

$N(A)=\frac{4}{\pi A} \sqrt{1-\left(\frac{\delta}{\mathrm{A}}\right)^{2}}$
$\mathrm{A}<\delta$

$\mathrm{A}>\delta$ 


\section{Phase Plane Stability and Filter Design (continued)}

- Describing function relay representation is still a nonlinear system as describing function gain is dependent on input amplitude (A):

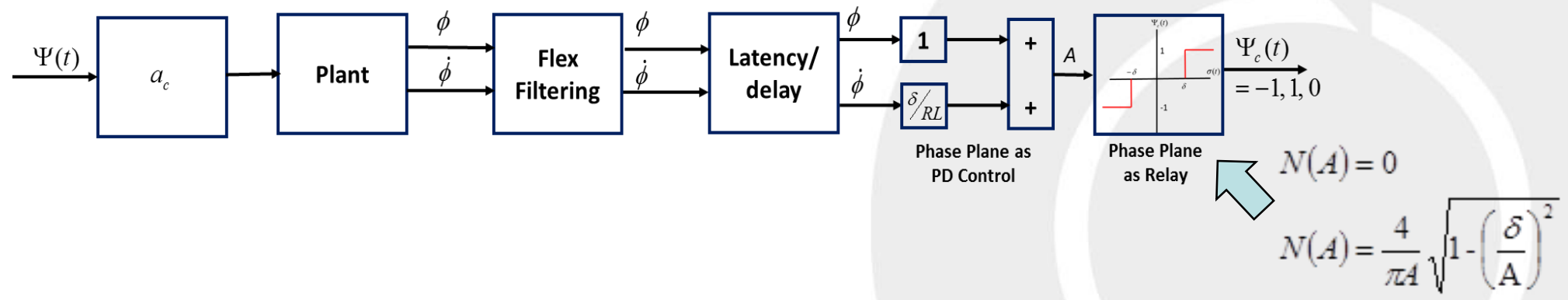

- Linearize system by deriving value of $A$ which maximizes the describing function $\left(A^{*}\right)$ :

$$
A^{*}=\sqrt{2} \delta \quad \rightarrow \quad \mathrm{N}\left(\mathrm{A}^{*}\right)=\frac{2}{\pi \delta}
$$

- Maximizing the describing function gain represents peak RCS control response to state error, which maximizes flex response to RCS firings (conservative approach). 


\section{Phase Plane Stability (continued)}

- Given a System:

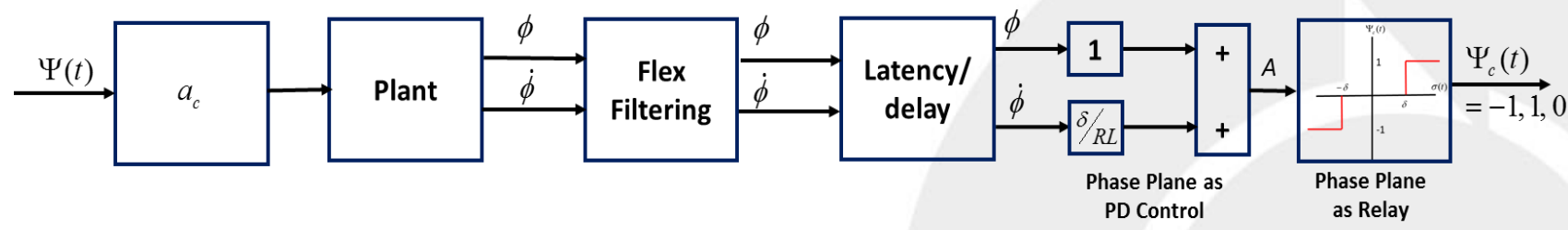

- Substitute the relay with a peak gain representation derived from the describing function:

$$
A^{*}=\sqrt{2} \delta \quad \rightarrow \quad \mathrm{N}\left(\mathrm{A}^{*}\right)=\frac{2}{\pi \delta}
$$

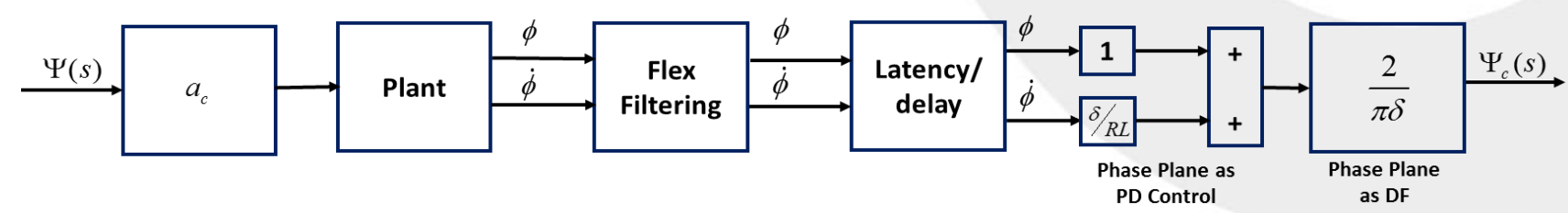

- The resulting derivation is a linear representation of phase control system. 


\section{Phase Plane Stability (continued)}

- Example. Model rigid body control and ideal latency:

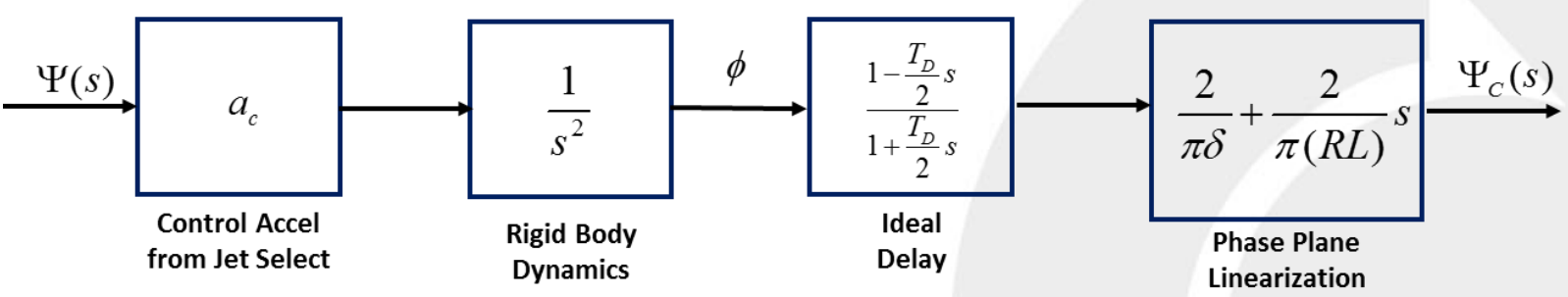

- The phase plane controller is a PD representation with the gains proportional to the phase plane deadzone (attitude and rate) limits.

- The closed loop transfer function is derived:

$$
\frac{\Psi_{c}(s)}{\Psi_{c}(s)}=\frac{\frac{2 a_{c}}{\pi}\left[-\frac{T_{D}}{2(R L)} s^{2}+\left(\frac{1}{R L}-\frac{T_{D}}{2 \delta}\right) s-\frac{1}{\delta}\right]}{\frac{T_{D}}{2} s^{3}+\left(1-\frac{a_{c} T_{D}}{\pi(R L)}\right) s^{2}+\frac{2 a_{c}}{\pi}\left(\frac{1}{R L}-\frac{T_{D}}{2 \delta}\right) s+\frac{2 a_{c}}{\pi \delta}}
$$

- And the necessary Condition for stability derived:

$$
\frac{1}{R L}-\frac{T_{D}}{\delta}-\frac{a_{c} T_{D}}{\pi(R L)^{2}}+\frac{a_{c} T_{D}^{2}}{2 \pi(R L) \delta}>0
$$




\section{Phase Plane Stability (continued)}

- Given the stability condition:

$$
\frac{1}{R L}-\frac{T_{D}}{\delta}-\frac{a_{c} T_{D}}{\pi(R L)^{2}}+\frac{a_{c} T_{D}^{2}}{2 \pi(R L) \delta}>0
$$

- Stability thresholds can be derived:

$$
\delta>\frac{T_{D}-\frac{a_{c} T_{D}^{2}}{2 \pi(R L)}}{\frac{1}{R L}-\frac{a_{c} T_{D}}{\pi(R L)^{2}}}
$$

$$
T_{D}<\frac{a_{c} \delta-\left(a_{c}{ }^{2} \delta^{2}+\pi^{2}(R L)^{4}\right)^{1 / 2}+\pi(R L)^{2}}{a_{c}(R L)}
$$

Smallest Deadband

Allowable Latency 


\section{Phase Plane Stability (continued)}

- Paper provides an example of how the stability condition maps to the RCS time domain simulation.

$$
T_{D}<\frac{a_{c} \delta-\left(a_{c}{ }^{2} \delta^{2}+\pi^{2}(R L)^{4}\right)^{1 / 2}+\pi(R L)^{2}}{a_{c}(R L)}
$$

Allowable Latency
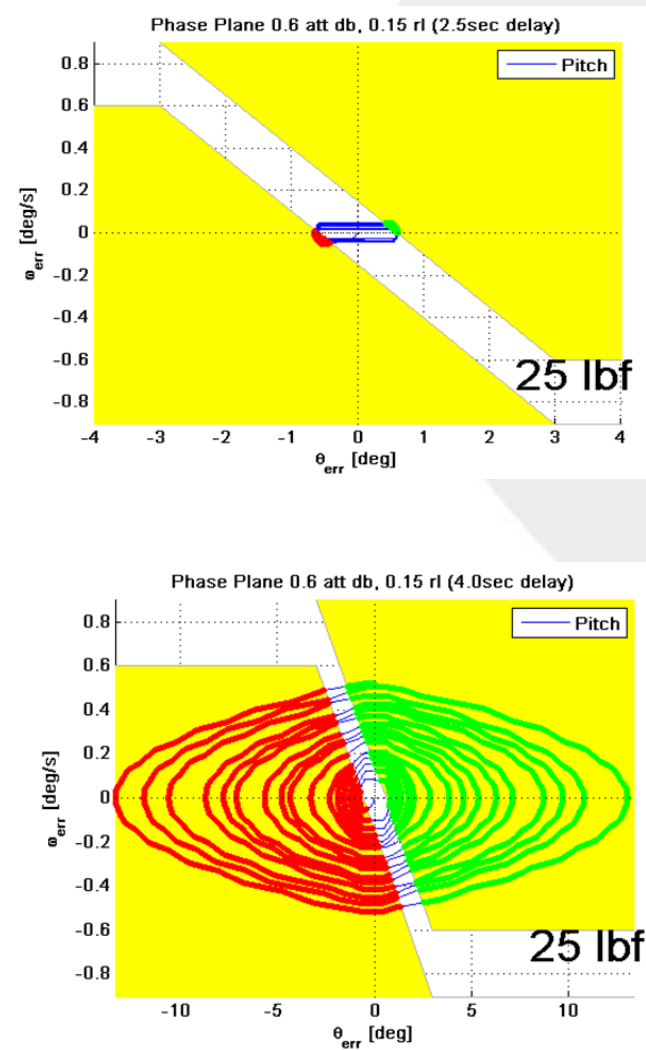
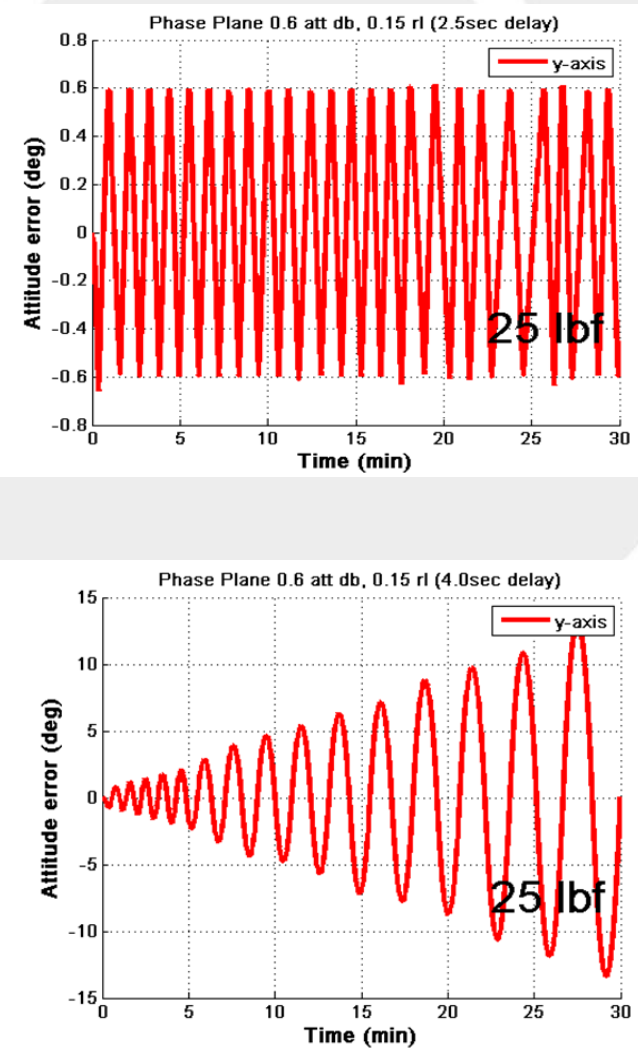


\section{Key RCS Filter Design Principles}

- Paper provides key filter Design Principles for RCS:

- Key Filter Design 1: Rigid body Stability

- Key Filter Design 2: Flex Gain Margins

- Key Filter Design Principal 3: Minimizing Filter Induced Lag

- Key Filter Design Principal 4: Feed Forward during Thruster Firings 


\section{Key Filter Design 2: Flex Gain Margins}

- Flex body dynamics can drive an RCS unstable.

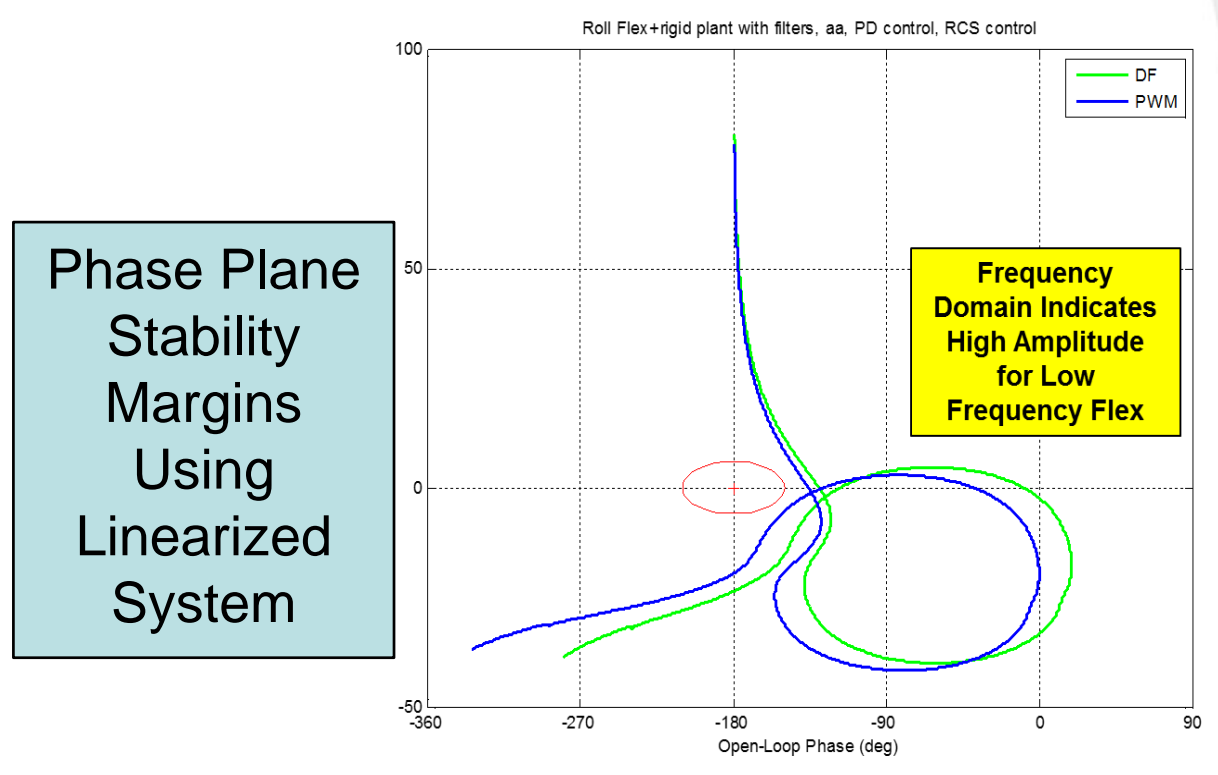

Phase Plane Flex Instability
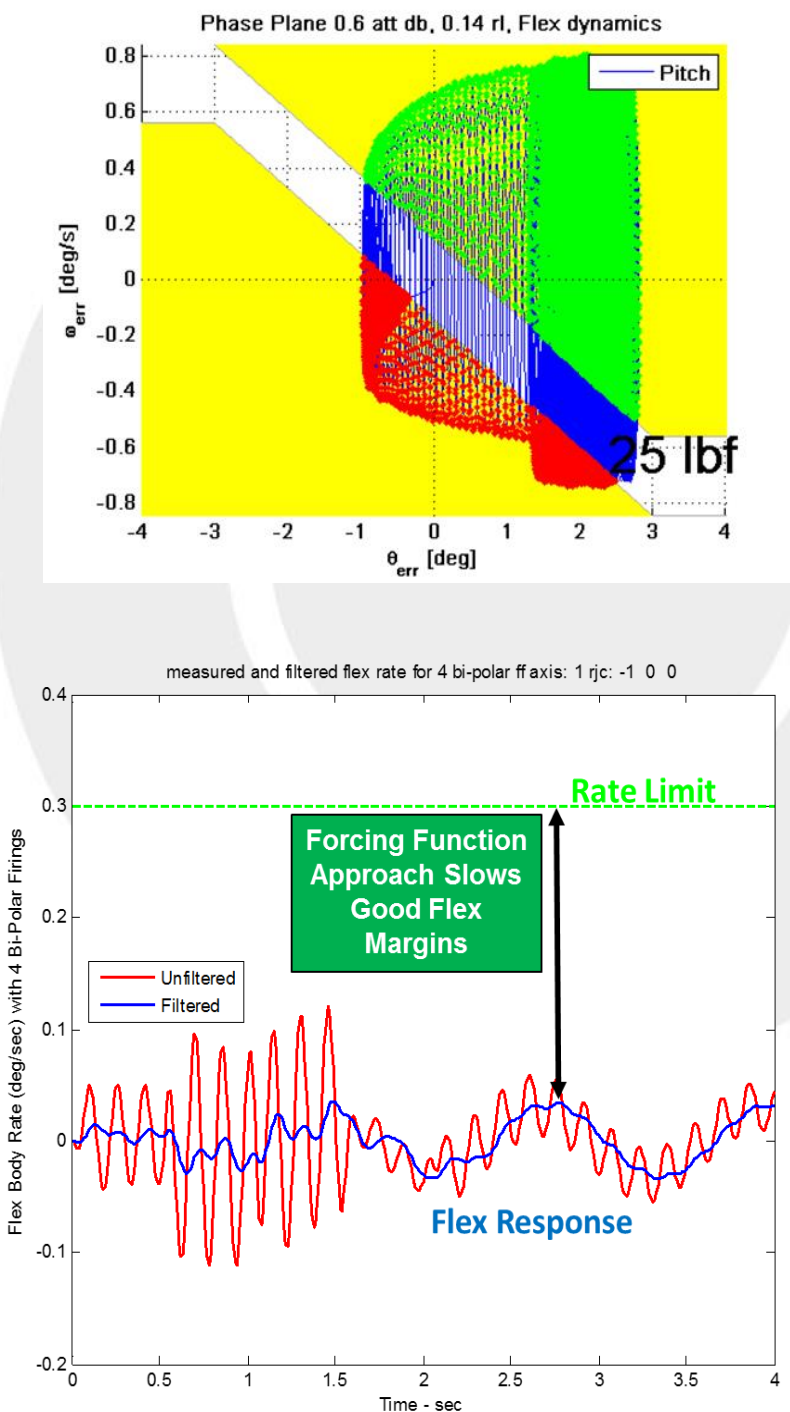

Phase Plane Stability Margins Shuttle Forcing Function Approach 


\section{OAIAA}

Shaping the Future of Aerospace

\section{Key Filter Design Principal 3: Minimizing Filter Induced Lag}

- Filter induced lag can result in a RCS limit cycle instability.
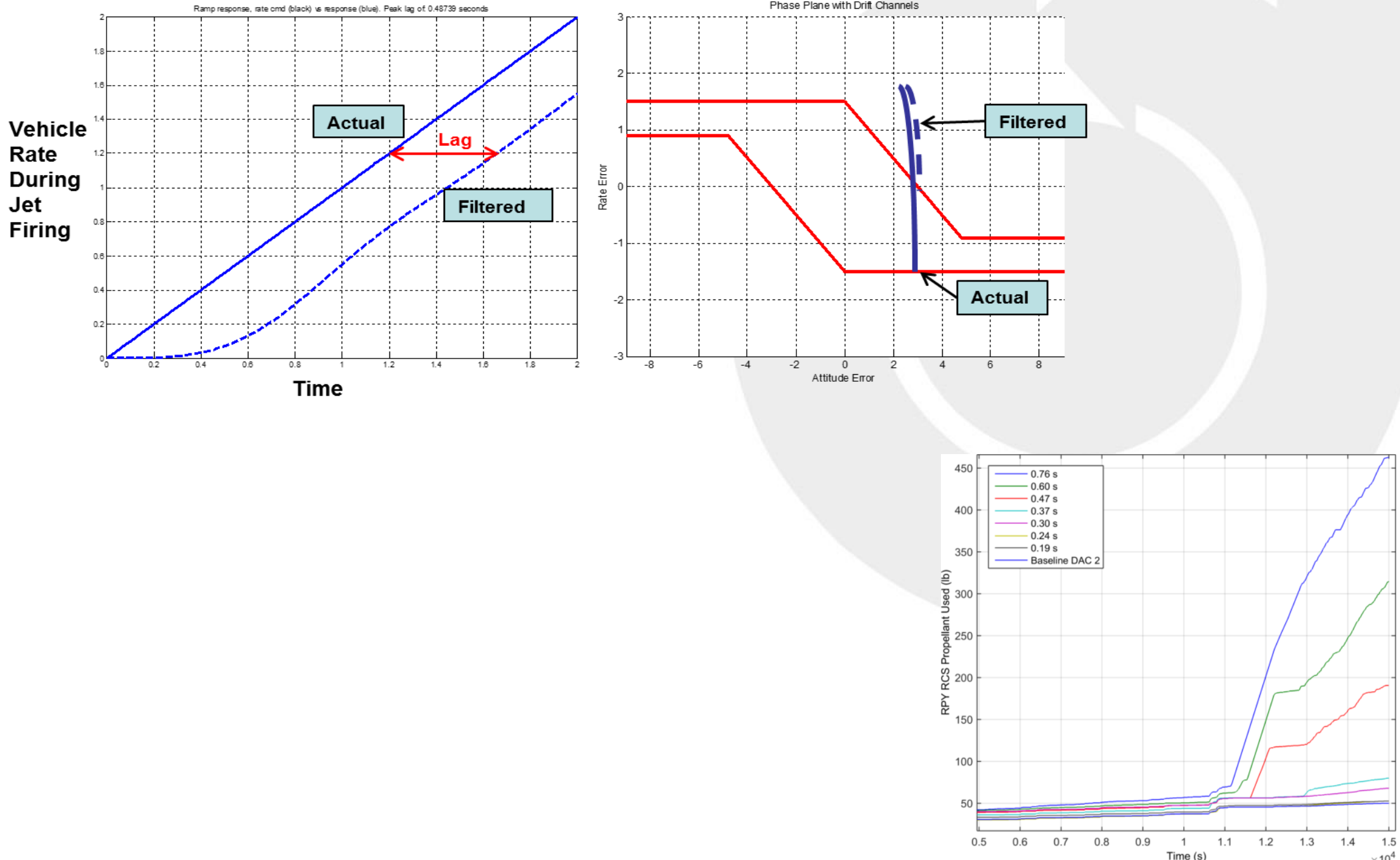


\section{RCS Jet Selection}

- Paper Addresses multiple RCS jet selection approaches:

- Table look-up.

- Algorithms that accommodate mass property changes.

- Fuel Optimal Jet Select.

- Command preshaping to avoid structural excitation.

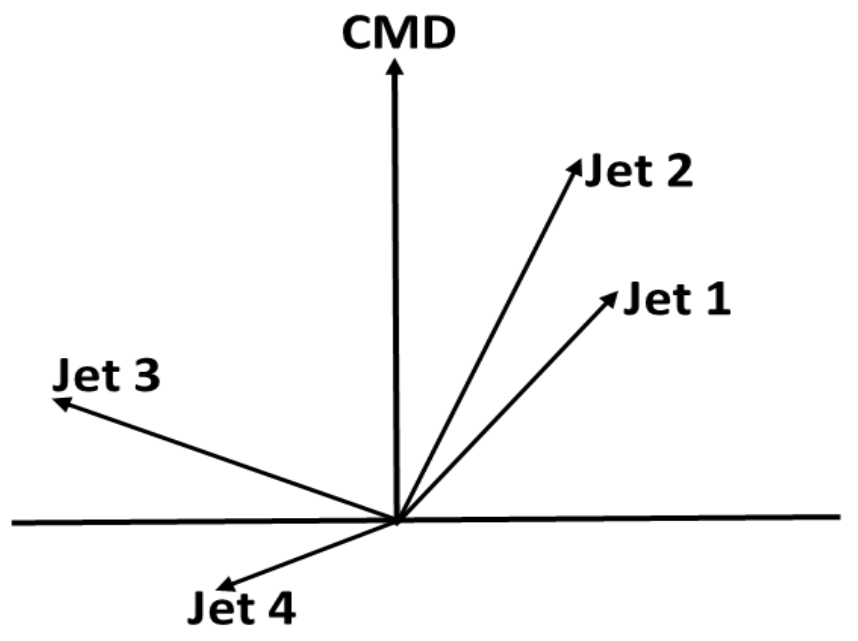

Two Space Shuttle Jet Select Algorithms

Dot Product: Would select jets 1 and 2

Minimum Angle: Would select jets 2 and 4 


\section{RCS Maneuvering/Steering Algorithms}

\section{- Paper Addresses multiple RCS maneuvering/steering approaches:}

- Eigen Axis Maneuvers.

- Torque-Free Maneuvers (Russian MIR).

- Steering Formulation.

- Fuel Optimal (Space Station "Zero Prop Maneuver").
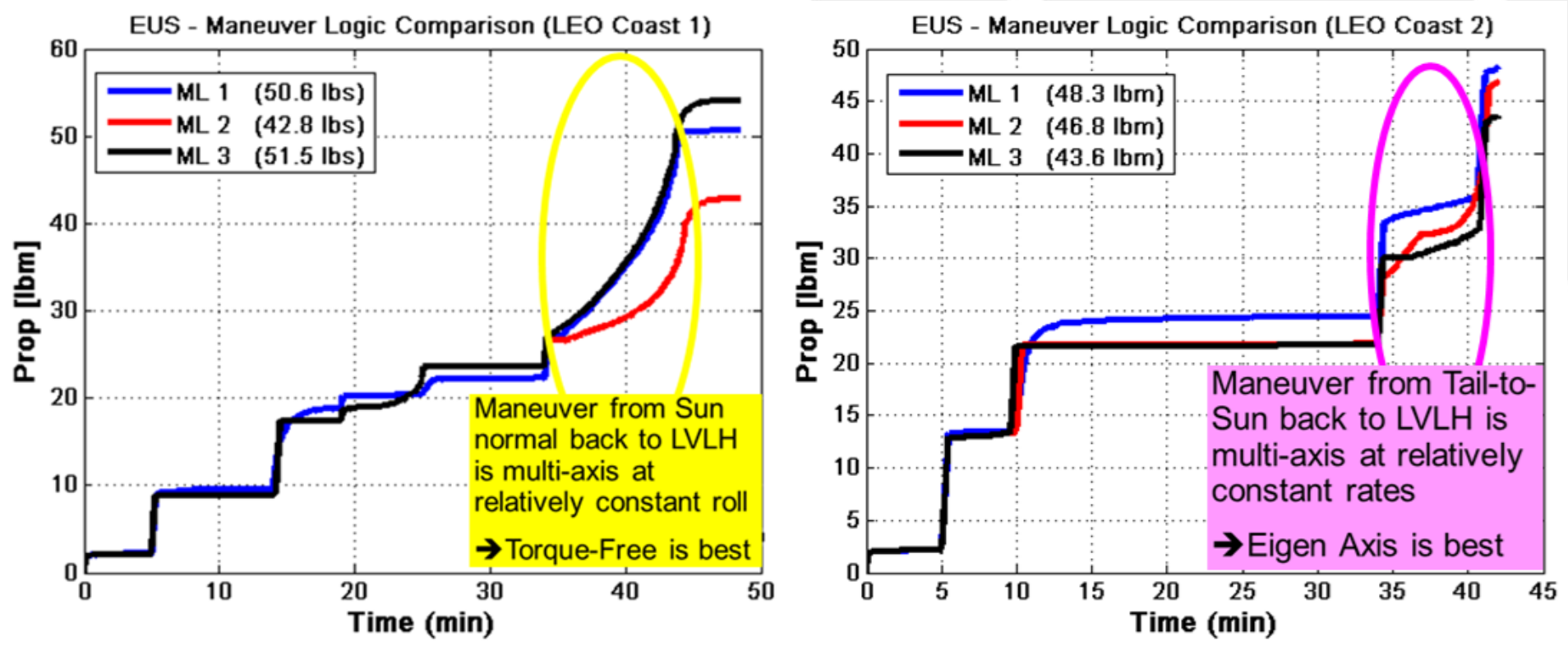


\section{Thruster Hardware Specifications}

- Discusses Shuttle RCS hardware design/control criteria:

- Control authority must exceed all known disturbances by a factor of two.

$$
\hat{T}_{C}>2 *\left|\max (-\hat{\omega} x \hat{J} \hat{\omega})+\max \left(\hat{T}_{e x t}\right)\right|
$$




\section{OJIAa}

Shaping the Future of Aerospace 